\title{
Medical Image of the Week: Bilateral Atrial Appendage Thrombi
}
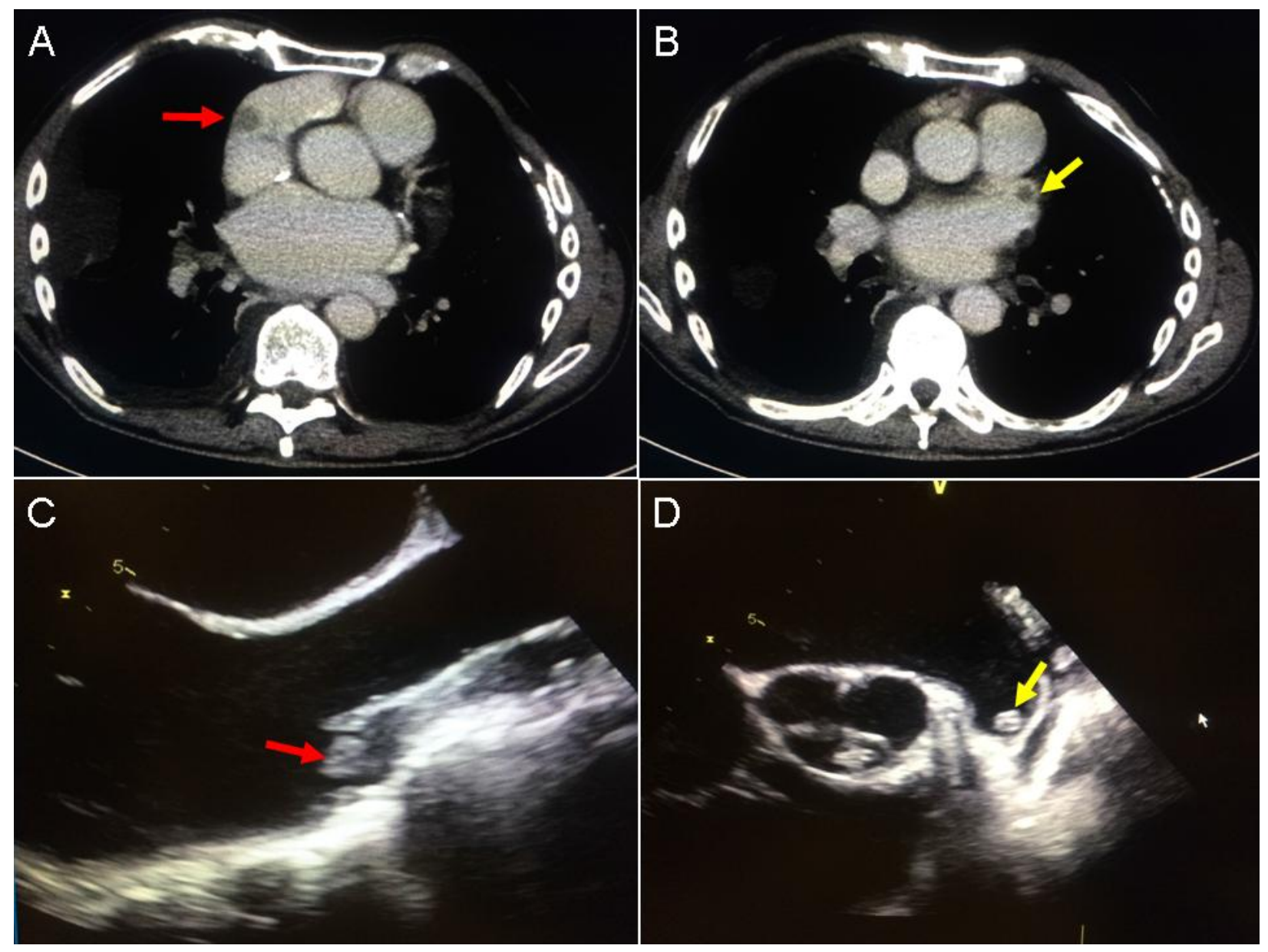

Figure 1. Panel A: Right atrial appendage (RAA) thrombus (red arrow) on chest computerized tomorgraphy angiogram (CTA). Panel B: Left atrial appendage (LAA)

thrombus (yellow arrow) on chest CTA. Panel C: RAA thrombus (red arrow) on transesophageal echocardiography (TEE). Panel D: LAA thrombus (yellow arrow) on TEE.

A 63-year-old man with a past history significant for hypertension, low back pain and polysubstance abuse (tobacco and marijuana) presented with shortness of breath and hemoptysis for the last 8 days prior to admission. His initial exam showed elevated jugular venous pressure and bilateral basal crackles with reduced air entry on the right lower lung zone.

The patient was found to be in atrial fibrillation with a rapid ventricular response. His initial chest X-ray showed a moderate right-sided pleural effusion. Immediate bedside echo was concerning for bilateral ventricular dysfunction with concerns of right-sided heart pressure and volume overload. A chest CT angiogram was obtained and showed acute lower lobe pulmonary embolism, with possible distal infarct, moderate right sided 
pleural effusion, and filling defects in both atrial appendages concerning for thrombi (Figure 1, Panels A \& B).

The patient was started on therapeutic anticoagulation and underwent therapeutic thoracentesis, gentle diuresis, and rate control for his atrial fibrillation. A few days later, a trans-esophageal echo confirmed the bilateral atrial thrombi (Figure 1, Panels C \& D).

Huthayfa Ateeli MBBS ${ }^{1}$, Andrew Kovoor MD ${ }^{1}$, Hem Desai MBBS ${ }^{1}$, Alana Stubbs MD², Tam Nguyen $\mathrm{MD}^{3}$

${ }^{1}$ Department of Medicine, ${ }^{2}$ Radiology Department, and ${ }^{3}$ Cardiology Division University of Arizona and Southern Arizona VA Health Care System

Tucson, AZ

\section{References}

1. Kim YY, Klein AL, Halliburton SS, Popovic ZB, Kuzmiak SA, Sola S, Garcia MJ, Schoenhagen $P$, Natale A, Desai MY. Left atrial appendage filling defects identified by multidetector computed tomography in patients undergoing radiofrequency pulmonary vein antral isolation: a comparison with transesophageal echocardiography. Am Heart J. 2007;154(6):1199-205. [CrossRef] [PubMed]

2. Shapiro MD, Neilan TG, Jassal DS, Samy B, Nasir K, Hoffmann U, Sarwar A, Butler J, Brady TJ, Cury RC. Multidetector computed tomography for the detection of left atrial appendage thrombus: a comparative study with transesophageal echocardiography. J Comput Assist Tomogr. 2007;31(6):905-9. [CrossRef] [PubMed] 